\title{
Evaluation of complications of PTBD using Hull's Risk Stratification Index
}

Prabhat Jha, Paleswan Lakhey

Department of Surgery, IOM, TUTH, maharajgunj, Kathmandu

Correspondence: Dr Prabhat Jha, MS Third year resident, Department of Surgery, IOM, TUTH, maharajgunj, Kathmandu

Email- prabhatkaushaljha@gmail.com

\begin{abstract}
Introduction: Obstructive jaundice is a common surgical problem. Percutaneous transhepatic biliary drainage (PTBD) is one of the effective treatments in the management of biliary obstruction. Hull Risk stratification index has been proposed to identify which patients are more likely to suffer from immediate and early complications and procedural mortality.
\end{abstract}

Materials and Methods: This is a descriptive observational study conducted at the department of surgery TUTH over a period of 1 year between october 2014 and october 2015. Hull risk stratification index was used to predict the complications of the procedure.

Results: 79 patients who underwent PTBD were included during the study period of one year. Among them $39(49 \%)$ were male and $40(51 \%)$ were female. The median age of patients undergoing the procedure was 59yrs (range 35-90yrs). Twenty nine patients (36\%) were in the medium Hull risk group, 50 (64\%) of patients were in the low risk group and there were no patients in the high risk group.

Six patients (7.5\%) died after the procedure. Seven patients (8\%) developed sepsis following the procedure. One patient (1.2\%) developed hemobilia. Two patients (2.4\%) developed pneumonia following the procedure. The sensitivity, specificity, positive predictive value, negative predictive value of the index for complications of PTBD were $81 \%, 74 \%, 44 \%$ and $94 \%$ respectively. Patients with medium Hull's risk were 3 times more likely to develop complications of PTBD than those with low risk.

Conclusion: Hull's Risk Stratification Index can be utilized as a marker for predicting the early, immediate complications as well as procedure related mortality.

Key words: PTBD complications, Hull's Index 